\title{
Timbre Discrimination in Zebra Finch (Taeniopygia guttata) Song Syllables
}

\author{
Jeffrey Cynx \\ Rockefeller University Field Research Center \\ for Ecology and Ethology
}

\author{
Heather Williams \\ Rockefeller University Field Research Center \\ for Ecology and Ethology and \\ Biology Department, Williams College
}

\author{
Fernando Nottebohm \\ Rockefeller University Field Research Center \\ for Ecology and Ethology
}

\begin{abstract}
Zebra finch (Taeniopygia guttata) songs include syllables of a fundamental frequency and harmonics. Individual harmonics in 1 syllable can be more or less emphasized. The functional role of this variability is unknown. These experiments provide evidence of how the phenomenon is perceived. We trained 12 male and female zebra finches on a go-no-go operant procedure to discriminate between 2 song syllables that varied only in the absence of the 2 nd or 5 th harmonic. Training involved many thousands of trials. Both sexes used the presence or absence of the 2nd harmonic as the sole discriminative cue. Females had more difficulty learning to perform the task when the presence of the 2nd harmonic was the go stimulus, which indicates that their use of the information was biased by stimulus-response contingencies. The results are discussed in terms of a broad strategy to understand how animals perceive sounds used in communication.
\end{abstract}

Harmonically structured syllables, in which frequency components stand in integer relations, are common in zebra finch songs and calls. The frequency components often vary in relative amplitude. For example, a song syllable's third harmonic may be suppressed $10 \mathrm{~dB}$ or more below any other harmonics (Figure 1, top panel). In a companion article we provided evidence of the learning, stability, neural control, and other production aspects of these song syllables (Williams, Cynx, \& Nottebohm, 1989). Because, as shown in that article, male zebra finches imitate specific patterns of suppressed harmonics, one may hypothesize that these patterns of harmonic suppression are perceived. However, we have little or no idea of how harmonic structures are perceived by songbirds. This article sets out to describe what sorts of harmonic amplitude differences can be discriminated and whether female zebra finches can perceive these differences. Together, the two articles provide an analysis of a functional link between the acoustical and perceptual abilities of a songbird.

Humans perceive variations in the amplitude levels of harmonics of spoken vowels (Pols, van der Kamp, \& Plomp,

Portions of this research were presented at the 14th meeting of the Society for Neuroscience, Toronto, Ontario, Canada, November 1988. The research was supported by National Institutes of Health Postdoctoral Training Grant MH15125 to Jeffrey Cynx, Public Health Service Award 17991 to Fernando Nottebohm, and Air Force Office of Scientific Research Award 86-0336 to Heather Williams. We also acknowledge the very generous support of the Mary Flagler Cary Charitable Trust and of Biomedical Research Support Grant SO7RR07065 awarded to Rockefeller University by the National Institutes of Health.

Correspondence concerning this article should be addressed to Jeffrey Cynx or Fernando Nottebohm, Rockefeller University Field Center for Ecology and Ethology, Tyrrel Road, Millbrook, New York 12545, or to Heather Williams, Biology Department, Williams College, Williamstown, Massachusetts 01267.
1969), musical instruments (Plomp, 1970, 1976), and sung vowels (Bloothooft \& Plomp, 1988). This human perceptual phenomenon is usually referred to as timbre (Plomp, 1976; Risset \& Wessel, 1982). Zebra finch song is divided into syllables, defined as continuous traces on the sonogram. Song appears to be learned and produced in syllabic units (Cynx, 1990; Price, 1979): We hypothesized that zebra finches could discriminate between birdsong syllables when the amplitude levels of individual harmonics varied. We therefore termed such a discrimination timbre solely on the basis of the acoustic similarity between the harmonic structures used in this article and those used in human research. We first show that changes in the timbre of zebra finch syllables are discriminable (Experiment 1 ). We then provide evidence of how the discrimination is controlled (Experiments 2 and 3). A final experiment shows that the naturally occurring frequency modulation in the stimuli was not used as a discriminative cue (Experiment 4).

\section{Experiment 1: Discrimination Training}

\section{Method}

Subjects. We trained and tested 6 male and 6 female adult (older than 90 days) zebra finches (Taeniopygia guttata), raised in Rockefeller University Field Center aviaries. All birds were experimentally naive and were housed in a laboratory aviary when not being trained or tested. The aviary diet consisted of mixed dry seed, mixed soft seed, and ground hard boiled eggs with shell. The birds were food deprived 4-6 hr before each experimental session. Mixed dry seed was used during sessions to reinforce discrimination behavior. Water and grit were available to birds at all times. Full-spectrum fluorescent lights in the aviary paralleled the natural photoperiod for New York state. Three sets of equal numbers of males and females were trained and tested at different times of the year. There were no seasonal 

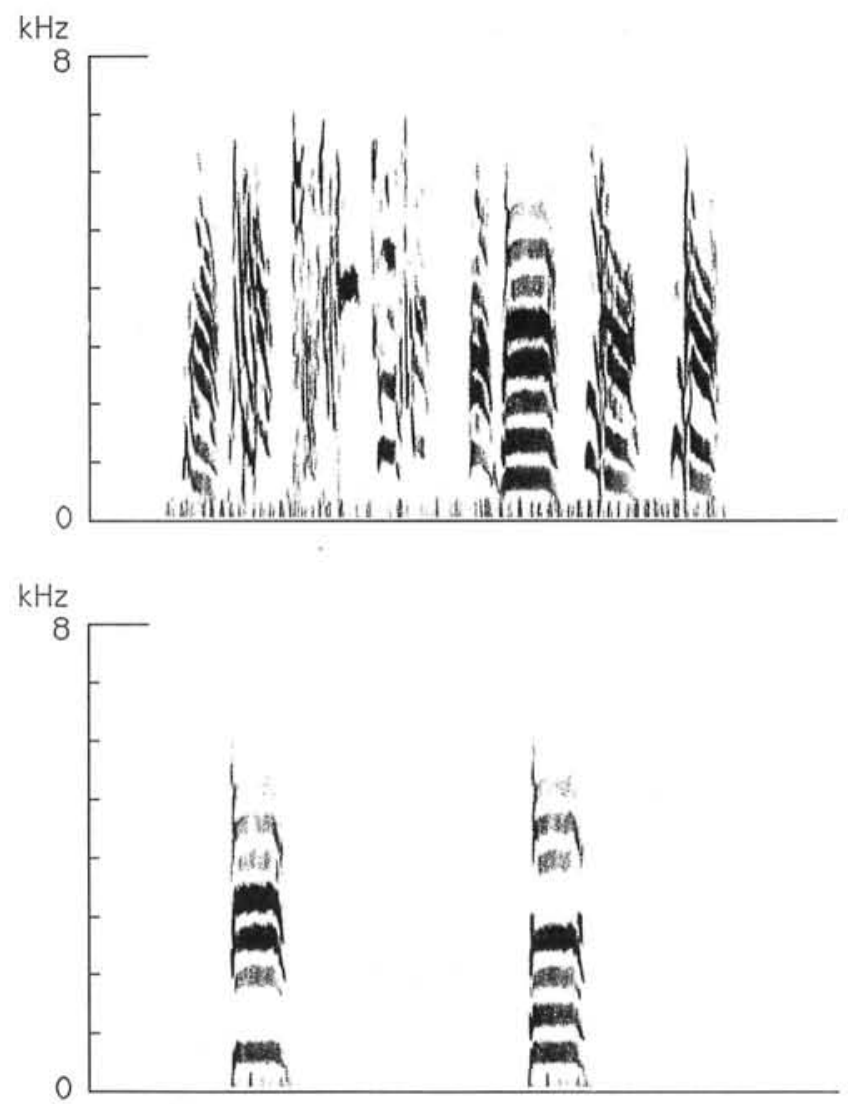

Figure 1. An example of zebra finch song (top panel) and the two training stimuli (bottom panel). (Horizontal lines mark off $1-\mathrm{kHz}$ frequency bands [Kay sonograph, Pine Brook, NJ; wide-band filter at $300 \mathrm{~Hz}$, without use of $\mathrm{Hi}$-shape filter]. The $\mathrm{x}$ axis represents time. The training stimuli are each approximately $100 \mathrm{~ms}$ in duration and were edited from one syllable of the song in the top panel. On the basis of a power spectrum of at least $12.8 \mathrm{~ms}$ of an unmodulated segment, Harmonics 1.9 in the training stimuli had the following amplitude levels in relation to the first harmonic: $0,+1,0,+3,+5$, $-1,-1,-3$, and $-9 \mathrm{~dB}$. The two training stimuli were built by attenuating either the second and fifth harmonics by $72 \mathrm{~dB}$, the maximum attenuation for a 12-bit signal. Recordings from the test chambers showed attenuation of at least $25 \mathrm{~dB}$ for these harmonics. The overall amplitudes for training stimuli were set at $72 \mathrm{~dB}$ SPL.)

effects in this song syllable discrimination (but see Cynx \& Nottebohm, 1990a 1990b, for seasonal effects with full song discriminations).

Behavioral apparatus. Six identical operant stations were used. Test cages were closed cylinders of $1.8-\mathrm{cm}$ wire mesh that measured $20 \mathrm{~cm}$ in diameter and $33 \mathrm{~cm}$ in length. One end of each cylinder was mounted on a $20 \times 20 \mathrm{~cm}$ aluminum panel that held two food magazines (dispensers with goal lights) $10 \mathrm{~cm}$ apart. Only the right food magazines were used in experiments reported in this article. Speakers (10 cm in diameter; Model $81-10 \mathrm{H}, \mathrm{JBL}$, Northridge, CA) were centered above the food dispensers so that they faced the birds.

Perch hopping was the operant behavior. Aluminum observation perches were placed parallel to the operant panels, $17 \mathrm{~cm}$ in front of the speakers. They contained $2.5-\mathrm{cm}$ infrared beam detector zones. Hopping elsewhere on the aluminum perches had no experimental consequences. Similarly equipped response perches were placed at the entrances to the food dispensers. A wooden perch placed $27 \mathrm{~cm}$ back from the aluminum panels allowed for noncontingent perch hopping.

The test cages were placed in separate sound attenuation chambers (Industrial Acoustics, Bronx, NY) with internal dimensions of $40 \mathrm{~cm}$ wide $\times 36 \mathrm{~cm}$ high $\times 59 \mathrm{~cm}$ long. Twenty-five-watt light bulbs mounted in the ceiling fixtures provided illumination. All inside surfaces of the chambers were lined with $1.27-\mathrm{cm}$ embossed acoustic foam. Behavior was observed through one-way glass windows and speaker monitors. Stimulus presentation, experimental contingencies, and data collection were controlled on line by an IBM (Armonk, NY) AT microcomputer.

Acoustical apparatus. Three analog boards (Models 2801 or 2821, Data Translation, Marlboro, MA) digitized and produced the song syllables (sampling rate of $20 \mathrm{kHz}$ ). Input was band-pass filtered $(0.2-$ $10.0 \mathrm{kHz}$ ) before being digitized. Output went to one channel of one of three power amplifiers (Model 85, BGW Systems, Hawthorne, $\mathrm{CA}$ ), then to one of six band-pass filters, and finally to the speakers.

Stimuli. The song syllable used in all four experiments was edited from the song of a male zebra finch that was not used in these experiments (Figure 1, top panel). The initial recording was made with a cassette recorder (Model PMD 221, Marantz, Chatsworth, $\mathrm{CA})$, digitized, and then edited and resynthesized with a fast Fourier transform (FFT) editing program (Zoloth, Dooling, Miller, \& Peters, 1980 ) designed by Robert J. Dooling to run on a microcomputer. The second harmonic was effectively removed from one digitized copy and the fifth harmonic from a second copy by attenuating those portions of the signal at least $25 \mathrm{~dB}$ (Figure 1, bottom panel). The decision to remove these specific harmonics was based on several considerations. First, we did not want to remove the first harmonic, or fundamental frequency, as that would raise questions of periodic versus spectral pitch perception (cf. Cynx \& Shapiro, 1986). Similar questions would be raised by removal of harmonics that are integer multiples of each other, for example, the second and fourth harmonics. Second, we wanted to choose two harmonics that were not adjacent but were roughly equal in amplitude. The power spectrum showed this to be so for the second to fifth harmonics. Third, we wanted one harmonic (the second) to be near the frequency band we had identified as containing many of the naturally occurring instances of suppressed harmonics (Williams et al., 1989). Finally, naturally occurring instances could have been used to meet these criteria, but the artificial creation of both stimuli from the same syllable provided us with greater control over acoustic features.

The first harmonic had a center frequency of $615 \mathrm{~Hz}$, the second harmonic, $1230 \mathrm{~Hz}$, and the fifth harmonic, $3075 \mathrm{~Hz}$, as measured on the sonogram and an FFT-based power spectrum; by these criteria, the syllable consisted of a stack of harmonics. The power spectrum showed intensity values of 52-61 dB SPL for each of the first nine harmonics. An audiogram for a zebra finch (Okanoya \& Dooling, 1987) shows thresholds fairly flat (less than 5-dB variation) in the frequency range between the second and fifth harmonics and well below the sound levels of individual harmonics used here.

The difference between the two stimuli is subtle, at least to human ears. We perceived a pitch at the first harmonic for both stimuli and a slight change in timbre between stimuli.

Procedure. The zebra finches were first trained to eat from the food magazine. The food dispenser and goal light were automatically turned on for $20 \mathrm{~s}$ every $3 \mathrm{~min}$. Hopping to the center of the observation perch also activated the magazine for $20 \mathrm{~s}$. All subjects initiated this operant over 200 times within three sessions, during which the reinforcement interval was reduced in steps to $5 \mathrm{~s}$. At this point the automatic functioning of the magazine was halted, and a go-no-go procedure was instituted. A go response to the go stimulus during the 3-s go period produced access to food. A go response to the no-go stimulus turned off the houselight for a 15 -s timeout. A nogo response always resulted in the end of the trial after $3 \mathrm{~s}$. 
The syllable with the attenuated second harmonic was the go stimulus for 6 birds. The reverse contingencies held for the other 6 birds. Sexes were equally divided between groups. The probability of a go or no-go trial was equal and random. The reinforcement interval was reduced to $1.7 \mathrm{~s}$ over 8-12 sessions. Each single, daily session lasted 3-6 hr.

Treatment of data. Acquisition was measured by recording the response times in both go and no-go trials. A criterion of a $0.5-\mathrm{s}$ positive difference between no-go and go daily means for three consecutive sessions was chosen as the acquisition criterion. This generally coincided with the birds' correct discrimination of $60 \%-$ $70 \%$ of the trials.

\section{Results}

It took many thousands of trials (and weeks of daily sessions) for the zebra finches to achieve criterion. Eleven of the 12 birds acquired the discrimination. Sex differences were correlated with the stimulus-response contingencies. Females had more difficulty learning to perform the task (and in one case failed) when the presence of the second harmonic was the go stimulus (Figure 2). A regression analysis was performed that excluded the 40,000 trials of the 12th bird. Regardless of this conservative approach, there was a significant interaction between the two categorical factors, sex and stimulus-response contingency, $F(1,7)=12.14, p<.01$. Inclusion of a degree of freedom for the twelfth bird would only have strengthened the effect.

These results establish that differences in harmonic emphasis, similar to those that appear in zebra finch song (Williams et al., 1989), are perceptible and can serve as discriminative cues. The differences between males and females may have been due to either the stimulus or the response properties of the task. In the first case the males and females may have attended to different stimulus features. In the second case they may have attended to the same features but shown different response dispositions. The first possibility has perceptual implications, the second motivational.

\section{Experiment 2: Manipulation of Individual Harmonics}

We next investigated what stimulus features were used as the discriminative cue in an effort to clarify the basis for the sex difference. We determined the discriminative cue for each bird by removing individual harmonics in order to reveal which harmonics controlled discrimination performance.

\section{Method}

Subjects. All the birds in Experiment 1 except the female that failed to acquire the discrimination were used in Experiment 2.

Stimuli. Nine test stimuli were synthesized with harmonics 1-9 attenuated 25-30 dB, respectively; two of these were the training stimuli. A 10th test stimulus had no harmonics attenuated (the original syllable, as shown in Figure 1, top panel).

Procedure. Reinforcement was lowered in steps to a random $65 \%$ of correct responses. The 10 test stimuli were randomly introduced during nonreinforced trials $(p=.15)$. Test stimuli were collected across two or three test sessions. A minimum of 15 observations were collected from each subject for each data point.
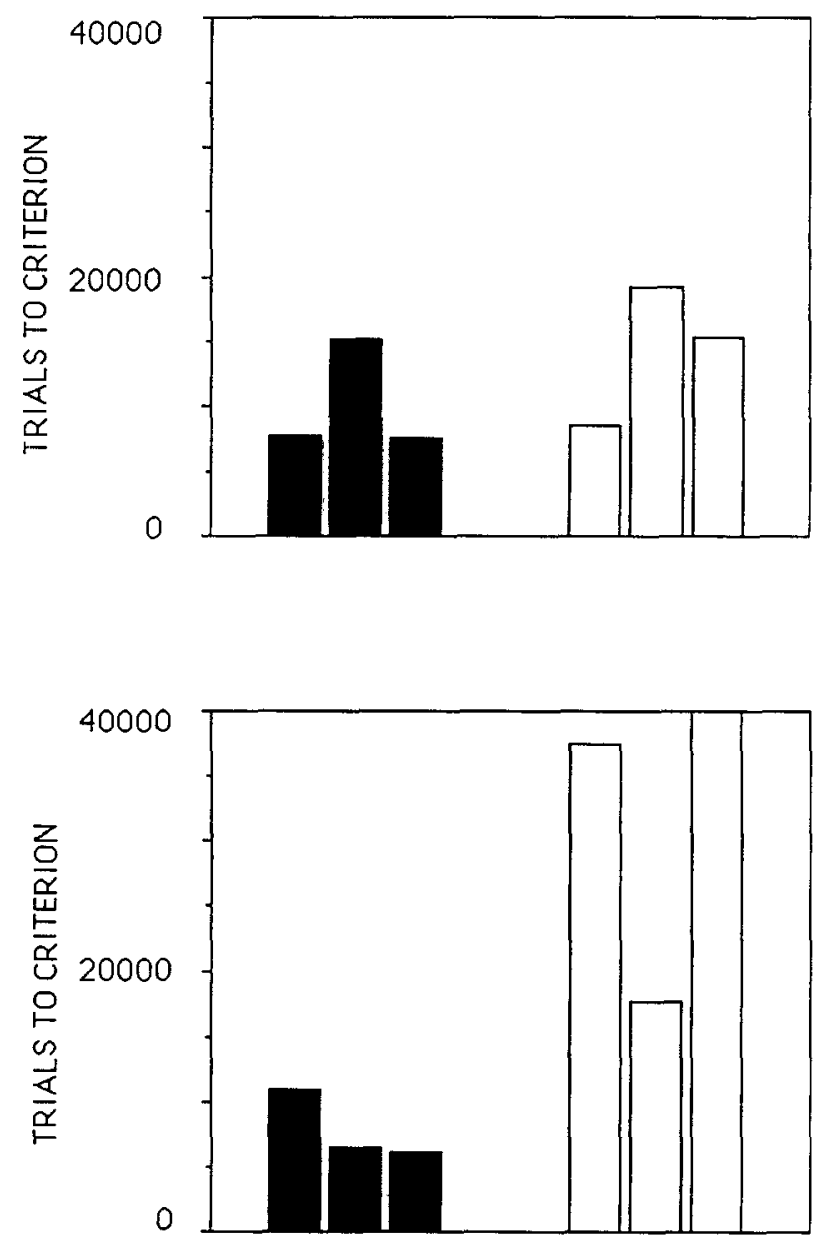

Figure 2. Trials to the 0.5-s criterion for birds trained to go when the second harmonic was missing (top panel) and for birds trained to go when the second harmonic was present. (Solid bars represent males, and open bars represent females.)

\section{Results}

The presence or absence of the second harmonic controlled discrimination performance for all birds, regardless of sex or the stimulus-response contingencies. Responses varied when the second harmonic was attenuated, whereas the presence or absence of other harmonics, including the fifth harmonic, had little or no effect. Some birds showed some generalization from the absence of the second harmonic to the absence of the first or third harmonics, but there was no indication that discrimination performance was controlled by the fifth harmonic (Figure 3).

\section{Experiment 3: Amplitude Reduction of the Second Harmonic}

In this experiment we determined the amplitude range over which the second harmonic controlled discrimination. The relative amplitudes of harmonic components may vary considerably, both within and between birds (Williams et al., 1989). If this variation has any communicative function, it must be perceptible. The training stimuli used up to this time 

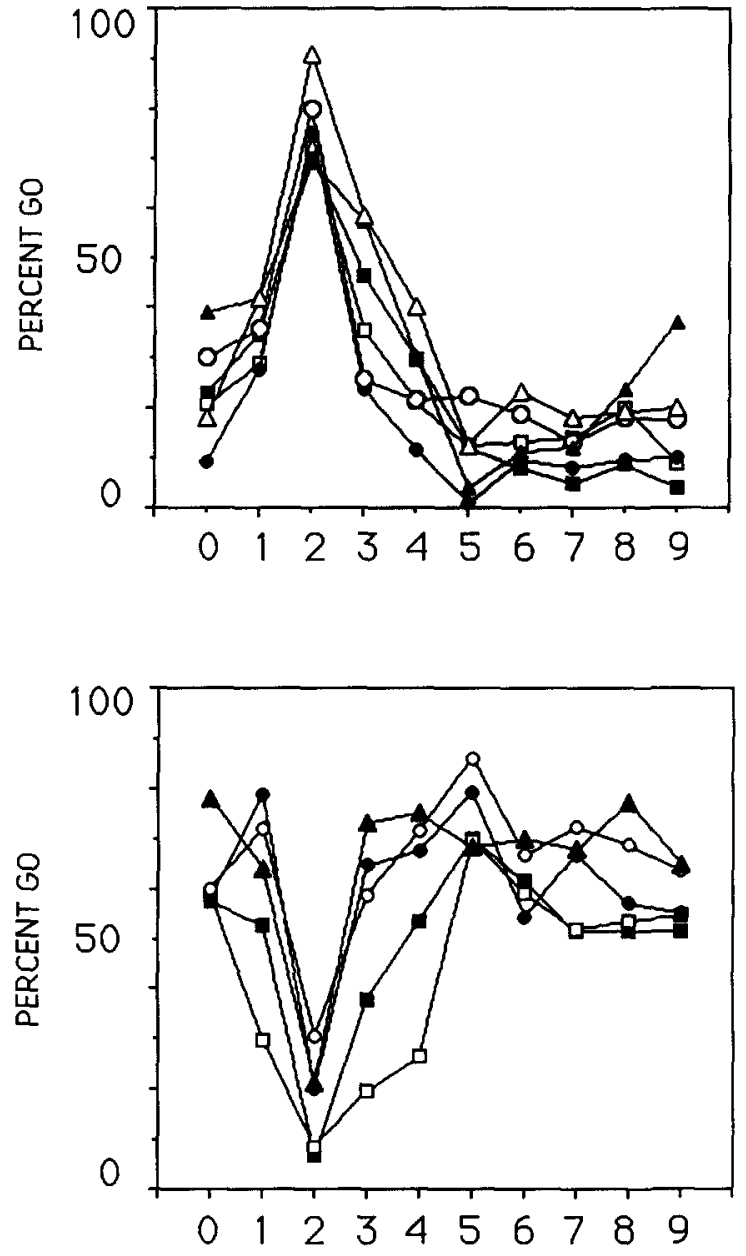

MISSING HARMONIC

Figure 3. Percentage of go responses when no harmonics were removed $(0)$ or when individual harmonics were removed (1-9) for birds trained to go when the second harmonic was missing (top panel) or present (bottom panel). (Solid points represent males, and open points represent females.)

had either the second or the fifth harmonic attenuated at least 25 dB. In Williams et al. (1989), zebra finches recognized amplitude differences in harmonics if they varied by at least $6 \mathrm{~dB}$. In this experiment we provide psychophysical evidence that a $6-\mathrm{dB}$ variation in the second harmonic of the training stimuli can be perceived.

\section{Method}

Subjects. The same subjects as in Experiment 2 were used.

Stimuli. We edited the song syllable (Figure 1, bottom panel) so the second harmonic varied from $2-15 \mathrm{~dB}$ below its original level $(-2,-5,-10,-12$, and $-15 \mathrm{~dB})$.

Procedure. The procedure was identical to Experiment 2.

\section{Results}

All birds detected a 5- to 10-dB reduction in the amplitude of the second harmonic. Many birds showed a change in response times with a $2-\mathrm{dB}$ reduction (Figure 4). Eight of the 11 birds showed a reversal in responding (crossing a $50 \%$ threshold) with a 5-dB attenuation of the second harmonic. Ten of the 11 birds showed a reversal in responding with a $10-\mathrm{dB}$ attenuation of the second harmonic. This indicates, on the basis of the discrimination procedure used here, that the variations in harmonic components examined in Williams et al. (1989) are perceptible.

\section{Experiment 4: Removal of Frequency Modulation}

The results of this control experiment ensured that stimulus control was tied to the frequency components of each harmonic alone. Both the beginning and the end of the syllable showed some frequency modulation (Figure 1, bottom panel). The birds might have attended to this property of the signal
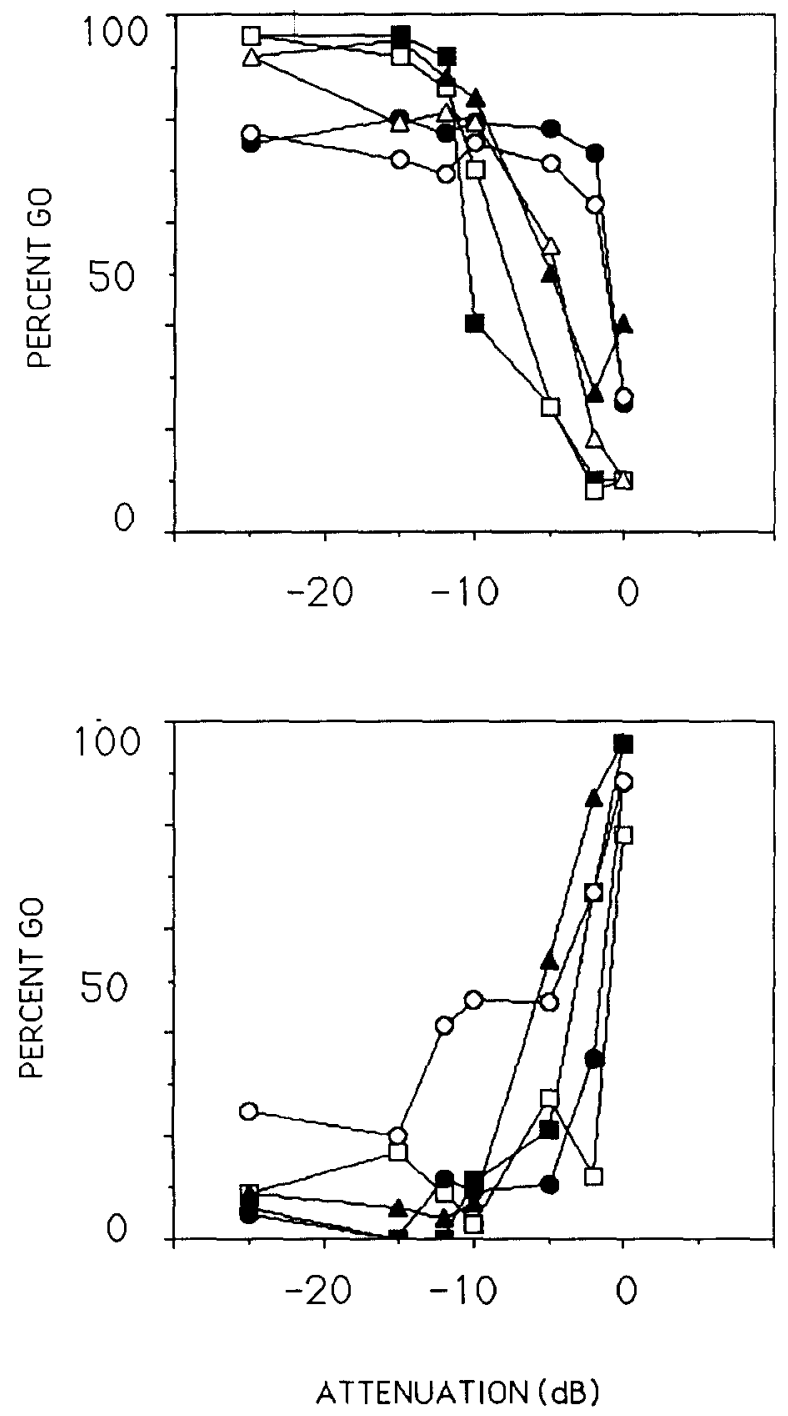

Figure 4. Percentages of go responses as the intensity of the second harmonic varied from -25 to $0 \mathrm{~dB}$ below its natural level for birds trained to go when the second harmonic was missing (top panel) or present (bottom panel). (Solid points represent males, and open points represent females.) 
that inadvertently occurred in the region of the second harmonic. We therefore removed time segments that contained the frequency modulation.

\section{Method}

Subjects. The same subjects as in Experiments 2 and 3 were used. Stimuli. The beginning or end of the training stimuli $(100 \mathrm{~ms}$ total length) was clipped by $10 \mathrm{~ms}$ to create two test stimuli. Five-ms cosine functions replaced the deleted segments of the test stimuli to prevent onset and offset clicks.

Procedure. The procedure was identical to Experiments 2 and 3.

\section{Results}

Removal of frequency modulation resulted in no noticeable differences between test and training stimuli, $F(1,10)<1, n s$, which indicates that discrimination performance was not controlled by the frequency modulation in the stimuli.

\section{Discussion}

Zebra finches can use differences in timbre to discriminate between song syllables. In the experiments reported in this article, the finches consistently used the presence or absence of the second harmonic as the discriminative cue, though in principle they could have just as well used the presence or absence of the fifth harmonic. The females' facility in acquiring the discrimination, but not the males', was dependent on stimulus-response contingencies. The results are discussed in regard to (a) avian harmonic perception, (b) sex differences, and (c) the neural substrates that govern perception of harmonically structured song elements.

\section{Avian Harmonic Perception}

The task in Experiment 2 could have been solved if the zebra finches categorized the test stimuli as high pitch or low pitch. Had they followed this strategy, then stimulus generalization ought not to have been symmetrical. The test stimulus with the first harmonic, or fundamental, missing would have produced a response that indicated it was categorized as similar to other test stimuli with lower harmonics missing. This was not the case (Figure 3). The results of Experiment 2 showed that the test stimulus with the first harmonic missing was categorized as similar to test stimuli with higher harmonics (3-9) missing. This indicates that the zebra finches assigned an absolute pitch to the frequency of the second harmonic, and the presence or absence of that pitch then governed responding.

In other research on the kinds of auditory cues used by songbirds, European starlings (Sturnus vulgaris) and a number of other songbirds also have shown a preference for using absolute pitch to solve discriminations, though they are capable of using relative pitch. This was true whether with pure tones (Cynx, Hulse, \& Polyzois, 1986; Page, Hulse, \& Cynx, 1989 ) or with harmonic structures (Cynx, 1985).

Songbirds' capacities for the use of true harmonic (relational) structures remain unknown, except for one report of periodicity pitch perception (Cynx \& Shapiro, 1986). No other evidence exists that songbirds perceive harmonic structures as harmonic, for example, that the class of harmonic sounds with missing second harmonics are perceived as similar. Furthermore, the ability to discriminate changes in timbre in no way indicates that a songbird's percept is similar to a human's. In fact, their use of absolute pitch suggests it is very different.

There is no obvious a priori reason why the zebra finches chose to use the second rather than the fifth harmonic as the discriminative cue. The second harmonic is closer to the frequency band that contains a disproportionate number of naturally occurring suppressed harmonics (Williams et al., 1989, Figure 9A and Table 2). We suggest two perceptual reasons for this production phenomenon. First, the presence or absence of lower-order harmonics may be more salient than that of higher harmonics-which may be related to the fact that frequency resolution in zebra finches is better at lower frequencies (Okanoya \& Dooling, 1987). Second, certain frequency regions may have special salience to the zebra finches, analogous to the special salience of formants and loci in human speech (Liberman, Cooper, Shankweiler, \& Studdert-Kennedy, 1967), and this may be related to central, rather than peripheral, processing.

\section{Sex Differences in Perception}

The acquisition results (Experiment 1) showed sex differences, whereas the determination of discriminative cues showed none (Experiment 2). This suggests the two sexes differed in how they used the stimuli, not in how they perceived them. Males were equally able to give go responses to the presence or absence of the second harmonic, if that was what they had been trained to do. Females found it more difficult than males to give go responses to the presence of the second harmonic. Sex differences in song development and production (Immelmann, 1969; Morris, 1954) and in the underlying neural substrate are well documented (Konishi \& Akutagawa, 1985; Nottebohm, 1980; Nottebohm \& Arnold, 1976; Williams, 1985), but do not give any clue as to why females show differences in learning to respond, given different stimulus-response contingencies. We had assumed that the second and fifth harmonics had an equal and neutral motivational valence. Perhaps the second harmonic, either as a harmonic or as an embedded frequency, has some communicative value for females in zebra finch song, so that the presence of this harmonic discourages approach. A second possibility, related more to associative learning theory than to motivation, is that the absence of the second harmonic is a hard cue for females to associate with a response approach. There is a precedent for a similar difficulty with other animals' learning to associate specific discrimination cues with specific responses (Konorski, 1967; Miller \& Bowie, 1982). Methods for measuring female zebra finch responses to song have recently been developed (Clark \& Nottebohm, 1990; Clayton \& Pröve, 1989), and the results in our experiments suggest that testing female choice with suppressed harmonics embedded in full song could be worthwhile.

\section{Neural Substrates of Harmonic Perception}

When a harmonic structure was played to guinea fowl (Numida meleagris), it was possible to detect the frequency 
components on tonotopic maps in Field $\mathrm{L}$, a structure in the auditory neostriatum (Scheich, Bonke, \& Langner, 1979). The pattern was the same as would be expected for a number of pure tone experiments, each pure tone equal to one harmonic. In songbirds, Field L projects directly to the higher vocal center (HVC; Kelley \& Nottebohm, 1979), a brain nucleus in the song control pathway essential for normal song production (Nottebohm, Stokes, \& Leonard, 1976). Single-unit data recorded from HVC in zebra finches show responses to auditory input (Katz \& Gurney, 1979; Williams \& Nottebohm, 1985). An unexpectedly high number of these units give their best responses to song syllables rich in harmonics. Interestingly, the attenuation of the higher harmonics does not diminish these unit responses, which, however, dampen on removal of certain lower (second and third) harmonics (Fortune \& Margoliash, 1989). The latter observation is in line with our results in Experiment 2 that show a greater weighting of the second than the fifth harmonic. However, it remains unclear whether the neurophysiological evidence that shows some HVC neurons are sensitive to the presence or absence of lower harmonics reflects the same process that governs the behavioral results. The ability of zebra finches to learn and then to perform these sorts of discrimination after lesions to the HVC may provide the crucial information.

\section{References}

Bloothooft, G., \& Plomp, R. (1988). The timbre of sung vowels. Journal of the Acoustical Society of America, 84, 847-860.

Clark, S. J., \& Nottebohm, F. (1990). Perception of birdsong by female zebra finches and canaries. Society for Neuroscience $A b$ stracts, 16, 1100.

Clayton, N., \& Pröve, E. (1989). Song discrimination in female zebra finches and bengalese finches. Animal Behaviour, 38, 352-354.

Cynx, J. (1985). Spectral and periodicity pitch perception in a species of songbird, the European starling (Sturnus vulgaris). Unpublished doctoral dissertation, Johns Hopkins University, Baltimore.

Cynx, J. (1990). Experimental determination of a unit of song production in the zebra finch (Taeniopygia guttata). Journal of Comparative Psychology, 104, 3-10.

Cynx, J., Hulse, S. H., \& Polyzois, S. (1986). A psychophysical measure of pitch discrimination loss resulting from a frequency range constraint in European starlings (Sturnus vulgaris). Journal of Experimental Psychology: Animal Behavior Processes, 12, 394402.

Cynx, J., \& Nottebohm, F. (1990a). Acquisition of a conspecific song discrimination is facilitated by testosterone implants in castrated zebra finches. Journal of Neuroscience Abstracts, 16,1099.

Cynx, J., \& Nottebohm, F. (1990b). Discrimination of conspecific songs by zebra finches (Taeniopygia guttata): Sex, season, and familiarity. Manuscript submitted for publication.

Cynx, J., \& Shapiro, M. (1986). Perception of missing fundamental by a species of songbird (Sturnus vulgaris). Journal of Comparative Psychology, 100, 356-360.

Fortune, E. S., \& Margoliash, D. (1989). Harmonic combinationsensitive neurons in the zebra finch. Society for Neuroscience Abstracts, 15, 617.

Immelmann, K. (1969). Song development in the zebra finch and other estrilid finches. In R. A. Hinde (Ed.), Bird vocalizations (pp. 61-74). Cambridge, England: Cambridge University Press.

Katz, L. C., \& Gurney, M. E. (1979). Auditory responses in the zebra finch's motor system for song. Brain Research, 221, 192-197.

Kelley, D. B., \& Nottebohm, F. N. (1979). Projections of the telen- cephalic nucleus-Field L-in the canary. Journal of Comparative Anatomy, 183, 455-470.

Konishi, M., \& Akutagawa, E. (1985). Neuronal growth, atrophy and death in a sexually dimorphic song nucleus in the zebra finch brain. Nature, 315, 145-147.

Konorski, J. (1967). Integrative activity of the brain. Chicago: University of Chicago Press.

Liberman, A. M., Cooper, F. S., Shankweiler, D. P., \& StuddertKennedy, M. (1967). Perception of the speech code. Psychological Review, 74, 431-461.

Miller, J. D., \& Bowie, C. A. (1982). Roles of the qualities and locations of stimuli and responses in simple associative learning. Pavlovian Journal of Biological Science, 17, 129-139.

Morris, D. (1954). The reproductive behaviour of the zebra finch (Poephila guttata) with special reference to pseudofemale behaviour and displacement activities. Behaviour, 6, 271-322.

Nottebohm, F. (1980). Brain pathways for vocal learning in birds: A review of the first 10 years. Progress in Psychobiology and Physiological Psychology, 9, 85-124.

Nottebohm, F., \& Arnold, A. (1976). Sexual dimorphism in vocal control areas of the songbird brain. Science, 194, 211-213

Nottebohm, F., Stokes, T. M., \& Leonard, C. M. (1976). Central control of song in the canary, Serinus canarius. Journal of Comparative Neurology, 165, 457-486.

Okanoya, K., \& Dooling, R. J. (1987). Hearing in passerine and psittacine birds: A comparative study of absolute and masked auditory thresholds. Journal of Comparative Psychology, 101, 715.

Page, S. C., Hulse, S. H., \& Cynx, J. (1989). Relative pitch perception in the European starling (Sturnus vulgaris): Further evidence for an elusive phenomenon. Journal of Experimental Psychology: Animal Behavior Processes, 15, 137-146.

Plomp, R. (1970). Timbre as a multi-dimensional attribute of complex tones. In R. Plomp \& G. F. Smoorenburg (Eds.), Frequency analysis and periodicity detection in hearing (pp. 397-411). Leiden, the Netherlands: Sijthoff.

Plomp, R. (1976). Aspects of tone sensation. San Diego, CA: Academic Press.

Pols, L. C. W., van der Kamp, T. J. Th., \& Plomp, R. (1969). Perceptual and physical space of vowel sounds. Journal of the Acoustical Society of America, 46, 458-467.

Price, P. H. (1979). Developmental determinants of structure in zebra finch song. Journal of Comparative and Physiological Psychology, 93, 260-277.

Risset, J.-C., \& Wessel, D. L. (1982). Exploration of timbre by analysis and synthesis. In D. Deutsch (Ed.), The psychology of music (pp. 26-58). San Diego, CA: Academic Press.

Scheich, H., Bonke, B. A., \& Langner, G. (1979). Functional organization of some auditory nuclei in the guinea fowl demonstrated by the 2-deoxyglucose technique. Cell Tissue Research, 204, 1727.

Williams, H. (1985). Sexual dimorphism of auditory activity in the zebra finch song system. Behavioral and Neural Biology, 44, 470484.

Williams, H., Cynx, J., \& Nottebohm, F. (1989). Timbre control in zebra finch (Taeniopygia guttata) song syllables. Journal of Comparative Psychology, 103, 366-380.

Williams, H., \& Nottebohm, F. (1985). Auditory responses in avian vocal motor neurons: A motor theory for song perception in birds. Science, 229, 279-282.

Zoloth, S. R., Dooling, R. J., Miller, R., \& Peters, S. (1980). A minicomputer system for the synthesis of animal vocalizations. Zeitschrift für Tierpsychologie, 54, 151-162.

Received February 6, 1990 Accepted February 7, 1990 